\title{
Therapierefraktäre rheumatoide Arthritis - eine Herausforderung
}

Trotz leitliniengerechter Therapien ist die Krankheitslast von Patienten mit persistierenden Zeichen und Symptomen einer rheumatoiden Arthritis (RA) hoch - die ökonomische Last für das Gesundheitssystem ebenfalls. Wann von einer "schwer zu behandelnden RA“ gesprochen werden sollte und welche Faktoren dabei mitspielen, war Thema beim EULAR-Kongress 2021.

Therapieziel in der Behandlung von Patienten mit rheumatoider Arthritis (RA) ist bekanntlich eine anhaltende Remission bzw. ein Zustand niedriger Krankheitsaktivität. Dies gelinge jedoch nicht in jedem Fall, so Prof. Dr. György Nagy, Semmelweis Universität, Budapest, Ungarn, im Rahmen der Sitzung zur EULAR-Definition der schwer zu behandelnden RA. In etwa 20 bis $30 \%$ der Fälle bleibt eine RA refraktär. Laut EULAR sollte dann von einer „Difficult-To-Treat RA“ (D2T-RA), also einer "schwer zu behandelnden RA", gesprochen werden. In Anlehnung an andere Disziplinen entschied sich die EULAR für diesen Begriff - statt für komplexe, refraktäre oder therapieresistente $\mathrm{RA}$. [1]

\section{Tab. 1 Kriterien für eine D2T-RA}

1. Es muss eine Behandlung gemäß den EULAR-Empfehlungen vorangegangen sein. Außerdem muss ein Versagen von mindestens zwei biologischen (b) bzw. zielgerichteten synthetischen (ts) DMARDs (Disease Modifying Anti-Rheumatic Drugs) mit unterschiedlichem Wirkmechanismus nach vorherigem Versagen einer Therapie mit einem konventionellen synthetischen (cs) DMARD vorliegen. Bei Kontraindikationen für csDMARDs entfällt diese Bedingung.

2. Es muss mindestens eines der folgenden fünf Kriterien erfüllt sein:

- Mindestens moderate Krankheitsaktivität, gemessen mit einem validierten Score, wie dem DAS28-ESR $>3,2$ oder CDAI $>10$

- Zeichen - darunter akute Phase Proteine und Bildgebung - und/oder Symptome einer aktiven Erkrankung

- Unmöglichkeit, die Steroid-Therapie auf unter 7,5 mg Prednison-Äquivalent pro Tag zu reduzieren

- Schnelle radiologische Progression, definiert als Veränderung des van der Heijde-modifizierten Sharp Scores um $\geq 5$ Punkte nach einem Jahr (mit/ohne Zeichen einer aktiven Erkrankung)

- RA-Symptome trotz nach obigen Standards gut kontrollierter Krankheit, die zu einer Einschränkung der Lebensqualität führen.

3. Das Management der Zeichen und/oder Symptome wird von Arzt und Patient als problematisch angesehen.
tsDMARDs bis zu immunologischen Mechanismen und fehlenderTherapieadhärenz. Unter den Komorbiditäten scheint die Fibromyalgie der wichtigste Faktor zu sein.

Anhand dieser Faktoren, die zu dem heterogenen Krankheitszustand einer D2T-RA beitragen, wurden drei Subgruppen definiert, allerdings auf Basis niedriger Fallzahlen: Die Gruppe der nicht-adhärenten, unzufriedenen Patienten, die von sich aus die Therapie intensivieren wollen, die Gruppe derer mit Schmerzsyndrom und Übergewicht und die Gruppe mit tatsächlich refraktärer RA, bei der der behandelnde Rheumatologe eine Intensivierung der Therapie wünscht.

\section{Literatur}

1. Nagy $G$ et al (2021) EULAR definition of difficult-to-treat rheumatoid arthritis. Annals Rheumatic Diseases 80:31-35

Hinweis des Verlags. Der Verlag bleibt in Hinblick auf geografische Zuordnungen und Gebietsbezeichnungen in veröffentlichten Karten und Institutsadressen neutral.

rheuma plus $2021 \cdot 20: 198$ https://doi.org/10.1007/s12688021-00451-w (c) Springer-Verlag GmbH Austria, ein Teil von Springer Nature 2021 